\title{
Gender disparities in weight loss surgery
}

\author{
Sherif Aly, Krista Hachey, Luise I. M. Pernar \\ Department of Surgery, Boston Medical Center, Boston University School of Medicine, Boston, MA 02118, USA.
}

Correspondence to: Dr. Luise I. M. Pernar, Department of Surgery, One Boston Medical Center Drive, Collamore 501, Boston, MA 02118, USA. E-mail: luise.pernar@bmc.org

How to cite this article: Aly S, Hachey K, Pernar LIM. Gender disparities in weight loss surgery. Mini-invasive Surg 2020;4:21. http://dx.doi.org/10.20517/2574-1225.2019.57

Received: 8 Dec 2019 First Decision: 25 Feb 2020 Revised: 4 Mar 2020 Accepted: 18 Mar 2020 Published: 10 Apr 2020

Science Editor: Wah Yang Copy Editor: Jing-Wen Zhang Production Editor: Tian Zhang

\begin{abstract}
Obesity is a growing epidemic affecting more than one third of the United States' population. It has detrimental effects on an individual's health and is associated with myriad negative outcomes including increased mortality. It also poses a substantial financial burden on the healthcare system. Weight loss surgery is an effective way of treating obesity with tremendous positive outcomes. Most patients who undergo bariatric surgery lose a significant amount of weight, reverse most of their comorbidities, and enjoy an improved quality of life. However, fewer than one percent of patients eligible for bariatric surgery actually undergo treatment. Furthermore, there exists a considerable gender disparity, with women comprising $80 \%$ of those patients who undergo bariatric surgery, despite equal obesity rates across genders. Many barriers exist between obese patients and weight loss surgery including misconceptions among patients and primary care providers regarding the perceived risk of surgery. This is in addition to numerous other psychosocial and cultural factors that may have contributed to and precipitated the existing gender imbalance. This review aims to highlight barriers to patients undergoing bariatric surgery and examine factors leading to the gender disparity that exists.
\end{abstract}

Keywords: Bariatric surgery, weight loss, gender disparity, obesity

\section{INTRODUCTION}

Obesity is a growing epidemic in the United States, affecting over one third of the population ${ }^{[1,2]}$. Numerous adverse health outcomes are associated with obesity, including type 2 diabetes, obstructive sleep apnea and cardiovascular disease, resulting in disability and substantial health care costs ${ }^{[3]}$. It is estimated that in 2013 obesity cost the healthcare system $\$ 116$ billion, of which $\$ 69$ billion were attributed to severe obesity ${ }^{[4]}$. In addition, obesity has been linked with a shortened life expectancy ${ }^{[5]}$, with approximately 122,000 deaths in the U.S. per year associated with obesity ${ }^{[6]}$. Weight loss surgery is often a last resort for patients, but may be

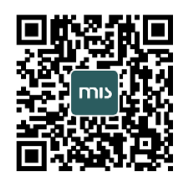


the only sustainable option for improved health outcomes, resulting in more than 50\% loss of excess body weight at 5 years postoperatively, significant improvements in chronic comorbid conditions, and improved life expectancy ${ }^{[6-8]}$.

Bariatric surgery is among the most common abdominal operation performed in the United States, with approximately 228,000 cases performed in $2017^{[9,10]}$. Minimally invasive laparoscopic weight loss procedures now account for over $90 \%$ of bariatric surgeries performed, with perioperative mortality rates declining to less than $0.1 \%$ over the past decade ${ }^{[3,9]}$. Patients referred for surgical evaluation at a bariatric program are typically seen by a multidisciplinary care team including a bariatric nurse, internist, psychologist or social worker, nutritionist, and surgeon to determine eligibility and optimize postoperative outcomes in selected patients $^{[11]}$.

Despite the tremendous benefits and well-documented safety of bariatric surgery, currently fewer than $1 \%$ of Americans medically eligible for weight loss surgery actually undergo treatment ${ }^{[12,13]}$. Referral patterns by primary care providers (PCP) seem to the biggest barrier to undergoing bariatric surgery. A recent metaanalysis suggested that patient and PCP concerns regarding complications and potential death from surgery were notable barriers to pursuing weight loss surgery ${ }^{[13]}$. However, providers who were more knowledgeable about bariatric surgery were more likely to refer their patients for consideration ${ }^{[13]}$. Additionally, Wee et al. ${ }^{[14]}$ reported that a recommendation by a PCP increased the likelihood that a patient would consider weight loss surgery by five times.

While the prevalence of obesity among men and women is similar in the general population, a substantial gender disparity has persisted over the past decade in bariatric surgery, with women comprising over $80 \%$ of patients undergoing weight loss surgery ${ }^{[2,15]}$. In addition to socioeconomic factors that influence access to bariatric surgical care, the complex interplay among gender, psychosocial, and cultural factors may pose an additional challenge to ensuring that all eligible patients, regardless of gender, are receptive to the option of weight loss surgery and have realistic expectations of outcomes after treatment.

We evaluated recent studies assessing demographic trends in bariatric surgery, as well as studies examining utilization and outcomes with a focus on gender disparities. We further evaluated and selected current literature based on the inclusion of themes relevant to gender-based differences in bariatric surgery care, including patient and provider perceptions of weight-loss surgery, provider referral patterns, and patient selection. We then sought to elucidate the sex-based differences in clinical and psychosocial outcomes after bariatric surgery. Our aim here is to highlight factors associated with the gender imbalance in the surgical treatment of obesity, review outcomes after surgery, and explore the critical opportunity for further collaboration between bariatric surgical specialists and PCPs to more effectively address gender-based disparities in obesity treatment.

\section{BARIATRIC SURGERY ELIGIBILITY AND UTILIZATION}

The National Institutes of Health (NIH) obesity management guidelines recommend bariatric weight loss surgery as a treatment for morbidly obese patients with a BMI $>40 \mathrm{~kg} / \mathrm{m}^{2}$ or a BMI of $35-40 \mathrm{~kg} / \mathrm{m}^{2}$ and obesity-related comorbidities ${ }^{[3,16]}$. Although obesity rates were once higher in female patients compared to male patients, these differences have gradually disappeared since the early $2000 \mathrm{~s}^{[17]}$. A review of the 20092010 National Health and Nutrition Examination Survey (NHANES) showed that the prevalence of obesity was similar among adult male and female patients with a prevalence of $35.5 \%$ and $35.8 \%$, respectively ${ }^{[18]}$. This suggests that the obesity rate may be climbing more rapidly in male than in female patients. However, the utilization of bariatric surgery is outstandingly lower among eligible men than women. In an analysis of the National Hospital Discharge Survey, which provides data on patients who have undergone bariatric surgery from 1999 to 2010, Mainous et al. ${ }^{[1]}$ found that, across racial groups, a significantly higher 
proportion of eligible women received bariatric surgery compared to eligible men. Another review of the Nationwide Inpatient Sample database from 2002 to 2011 showed similar results. Of the 810,999 patients who underwent bariatric surgery over that 10 -year period, only $19.3 \%$ were men ${ }^{[19]}$. These data imply that there is a gender-based disparity in the utilization of weight loss surgery among eligible patients.

Several studies demonstrate that a higher proportion of female bariatric surgery patients are younger (less than 45 years old) compared to their male surgical counterparts ${ }^{[1,15]}$. The increased proportion of younger women undergoing weight loss surgery may be due to strong cultural and social pressures to seek a thin body weight ideal resulting in higher patient request and referral for surgical evaluation ${ }^{[14,15,20]}$. Indeed, over $70 \%$ of referrals to bariatric surgical programs in the published literature are female and about half of the referrals are initiated by patients rather than by primary care physicians or other referring doctors ${ }^{[20-22]}$. These patterns suggest that patient-driven requests for evaluation at the primary care level are relevant to the gender imbalance seen among patients ultimately selected for surgical treatment. Additionally, bariatric surgery utilization by young women may reflect fertility issues as a result of obesity, resulting in surgical treatment to improve chances of a successful pregnancy and to reduce the risks of pregnancy-related complications ${ }^{[23,24]}$.

In contrast, social, cultural, and reproductive pressures to address weight perhaps provide less of an impetus for morbidly obese men to pursue surgical treatment earlier. Male patients typically present for surgery at an older age and with more comorbidities, including more than double the prevalence of coronary artery disease and history of myocardial infarction compared to morbidly obese women ${ }^{[1,15]}$. This delayed presentation for surgical treatment among men increases their risk of morbidity, disability, and mortality. In addition, among patients who initially attend a bariatric program for consideration of surgery, men are significantly more likely than women to drop out of the process without undergoing surgery $(\mathrm{OR}=0.527, P<0.001)^{[20]}$.

Although obesity rates are equal among genders, men are significantly less likely to be referred or undergo bariatric surgery. Understanding the gender-based differences in referral, program attrition, and utilization of bariatric surgical treatment are relevant to ensuring that high risk patients are adequately identified before potentially disabling and life-threatening comorbidities develop.

\section{GENDER-SPECIFIC WEIGHT PERCEPTIONS AND QUALITY OF LIFE}

The gender disparity among bariatric surgical patients may in part be explained by gender-based differences in perceptions of body weight and obesity-related quality of life, which may have an impact on the motivation to request evaluation for surgery, as well as follow through with treatment. In a study by Tsai et al. ${ }^{[25]}$, data from NHANES were used to evaluate gender-specific weight perception across increasing BMI. Compared with their female counterparts, overweight and obese men were less likely to have an accurate weight perception, weight dissatisfaction, and attempted weight loss. Some studies have found more pronounced gender-based difference among obese blacks compared to whites, suggesting that ethnicity and culture influence perceptions of ideal body weight ${ }^{[1]}$.

In a study of over 330 patients with a BMI of $35 \mathrm{~kg} / \mathrm{m}^{2}$ or higher recruited from primary care practices, Wee et al. ${ }^{[14]}$ assessed the relationship among obesity, perception of body weight, and patient quality of life via the Impact of Weight on Quality of Life-lite (IWQOL-lite) survey, which was specifically designed for use with obese individuals. IWQOL-lite examines domains including physical function, public distress, self-esteem, sexual life, and work. In this patient sample, women were disproportionately negatively affected by their weight compared to men, with statistically significantly lower Quality of Life (QOL) overall scores, as well as in the specific domains of weight-related social stigma, self-esteem, public distress, and physical functioning.

White et al. ${ }^{[26]}$ identified similar gendered patterns in obesity-related QOL using IWQOL-lite in a study of 512 individuals actively undergoing evaluation for bariatric surgery. Despite having the lowest overall 
BMI levels within the sample, white women had the most impaired QOL, and African American men had the least impairment. Within specific domains, black men (67\%) and white men (63\%) were more likely to report physical functioning deficits, compared to black women (51\%) and white women (46\%). Obesity related sexual limitations were identified as the most commonly reported impairment among both black women $(35 \%)$ and white women (24\%) compared to only $11 \%$ of white men and no black men.

These studies suggest that psychosocial distress associated with obesity is more strongly experienced by women and perhaps may explain part of the trend to presentation at a younger age, when social and cultural pressures are strongest. As Tsai et al. ${ }^{[25]}$ suggested, culturally accepted ideal male body types may permit a heavier weight without social detriment or decreased sense of self-esteem. However, it appears that detriments to physical agency brought on by morbid obesity and its comorbidities do have an impact on QOL for men and may be an important motivation for seeking treatment. Thus, a vital step in counseling patients for weight loss and consideration of bariatric surgery may involve helping patients identify morbid obesity as a serious health problem through its impact on aspects of day-to-day life that matter most to the individual, with sensitivity to gendered patterns of self-perception and coping with obesity-related impairments.

\section{GENDER-SPECIFIC PERCEPTIONS OF WEIGHT LOSS SURGERY}

In addition to gender-based differences in coping with morbid obesity and quality of life concerns, perceptions of bariatric surgery itself and motivations to pursue surgery may differ between men and women. Among 325 primary care patients with a BMI of $35 \mathrm{~kg} / \mathrm{m}^{2}$ or higher, Wee et al. ${ }^{[27]}$ explored demographic and quality of life factors affecting patient consideration of bariatric surgery and found that the majority of patients were aware of weight loss surgery, and overall $37 \%$ had ever seriously considered undergoing surgical treatment. Broken down by gender, $40 \%$ of women had ever considered surgery compared to $22 \%$ of men $(P<0.05)$. Only $35 \%$ of this patient cohort reported ever having received information about surgical weight loss options from a provider, with no gender differences. However, women were more likely to have received a physician recommendation for surgery compared to men $(22 \%$ vs. $14 \%, P<0.05)$. This is interesting given that nearly half of patients responded that they would seriously consider weight loss surgery if recommended by their provider, and this held true for both men and women. With regards to the other half, the perception of weight loss surgery as "too risky" was the most commonly reported deterrent to considering bariatric surgery across gender and ethnicity groups ${ }^{[27]}$. Both patient perceptions of risk associated with weight loss surgery as well as physician behaviors related to patient education and treatment recommendations influence individual and gender-specific consideration of surgical treatment options.

Patient motivations for pursuing weight loss surgery have been shown to differ by gender, echoing differences observed regarding self-perception of body weight and associated impairments in quality of life. In an Australian study of 208 participants approximately one year postoperatively after laparoscopic adjustable band placement, patients who reported distress related to appearance as a primary motivation to pursue weight loss surgery were more likely to have a lower presenting BMI and to be female $(P=0.03$ and $P<0.001$, respectively ${ }^{[28]}$. Additionally, patients who were motivated primarily by medical conditions were more likely to be men $(P=0.007)$, to be older, and to have hypertension or diabetes. Similarly, in a majority women (95\%) United States survey study of 44 patients evaluated for bariatric surgery, 84\% of participants noted psychosocial concerns as an extremely important motivating factor for surgery, although healthrelated reasons were also ranked by most as equally critical ${ }^{[29]}$. Patients expressed high expectations for sustained weight improvement on the order of about $80 \%$ of excess weight loss with surgical intervention. The authors noted that this degree of weight loss cannot always be achieved postoperatively and suggested that it is critical for patients to have more accurate weight loss expectations in order to achieve sustained satisfaction and improved outcomes after surgery. 
As men are underrepresented in the majority of bariatric surgical studies, Natvik et al. ${ }^{[30]}$ conducted a qualitative focus group study including 13 men who had previously undergone bariatric surgery to better understand their experience with surgical treatment. The men being interviewed reported that their initial misconceptions regarding weight loss surgery involved associating the treatment with "vanity, which they regard as valueless and shallow and did not relate to" ${ }^{\text {,[30] }}$. Prior to surgery, many men in the group had tried other weight loss options on their own, expressing that autonomy and independence were critical to addressing weight problems. The majority of men reported having suffered an acute illness such as stroke or heart attack, which brought up themes of powerlessness and an emerging realization of needing help for obesity, which they began to understand as a serious illness only after experiencing weight-related complications.

In this small cohort, evaluation for surgical weight loss treatment was frequently initiated by a healthcare professional or family member rather than by the men themselves. However, some men reported that the pursuit of weight loss surgery revived a sense of self-efficacy, which facilitated a commitment to treatment. While clearly a small study with limited generalizability and potential recall bias, this qualitative exploration of men's perceptions of bariatric surgery demonstrated that misconceptions of the purpose of bariatric surgery, that it is for body image rather than health, may be a significant barrier to appropriate treatment for men. The findings suggest that effective counseling of obese patients requires a gendered understanding of how patients individually relate their bodies and how that relationship is connected to what individuals value most in terms of physical and psychological health, as well as with respect to autonomy and personal agency.

\section{KNOWLEDGE GAP, PROVIDER REFERRAL, AND PATIENT SELECTION PATTERNS}

Although patients' perception and motivation to pursue weight loss surgery play a major role in access to bariatric surgery, the knowledge gap and increased perceived risk on behalf of providers and patients pose significant barriers between morbidly obese patients and surgical treatments. In a survey study of over 470 physicians, Avidor et al. ${ }^{[21]}$ reported that, among primary care physicians as well as other specialists in obstetrics and gynecology, cardiology, and endocrinology, most physicians had only moderate familiarity with the NIH morbid obesity management guidelines, safety of surgical options, and the long-term impact of surgery on weight and comorbidities. Additionally, the dominant reason for physician non-referral was provider lack of knowledge of a local bariatric surgeon, suggesting that surgical specialists need to improve outreach efforts and to expand surgical resources to underrepresented regions. Additionally, nearly half of gynecologists were unaware of published studies on the effects of bariatric surgery on restoring fertility in morbidly obese females. Furthermore, up to $35 \%$ of primary care practitioners surveyed have reported feeling unprepared to provide long-term medical care for post-surgical patients and less than half felt competent to manage medical complications of bariatric surgery ${ }^{[22]}$.

Additionally, perceptions of weight loss surgery as carrying increased risk further hinder access across genders. Funk et al. ${ }^{[31]}$ conducted focus groups with 16 PCPs in Wisconsin to better elucidate their perception of obesity and weight loss surgery. Interestingly, providers were primarily focused on obesity being a risk factor for disease instead of considering it a disease in and of itself. This is despite the American Medical Association resolution in 2013 characterizing obesity as a disease. Additionally, decision making by PCPs often under-prioritized treatment for obesity and over-emphasized risk of surgery. This study outlined several factors including PCPs wanting to "do no harm", and questioning the effectiveness of weight loss surgery as reasons for not referring patients ${ }^{[32]}$. However, the data argue against this, and repeatedly bariatric surgery has been shown to be equally safe as, and in some cases more safe than, other well accepted surgical procedures. Aminian et al. ${ }^{[33]}$ compared the safety of the laparoscopic Roux-en-Y gastric bypass (LRYGB) to seven other procedures in diabetic patients using NSQIP data between 2007 and 2012. The complication rate of LRYGB (3.4\%) was comparable to that of laparoscopic cholecystectomy (3.7\%) and laparoscopic hysterectomy (3.5\%), and significantly lower than that of total knee arthroplasty 
(16.7\%). This is interesting since obesity is a major risk factor for osteoarthritis, which in turn is the most common indication for a knee replacement. Similarly, LRYGB had a lower mortality rate than laparoscopic cholecystectomy and appendectomy ( $0.3 \%$ vs. $0.7 \%$ and $0.5 \%$, respectively). Future partnerships between bariatric surgeons and PCPs are crucial to increase knowledge about the safety of weight loss surgery.

Although referral patterns seem to be a larger barrier for access to weight loss surgery, patient selection by bariatric surgeons may also be implicated in the gender disparity. In a national survey study by Santry et al. ${ }^{[34]}$, patterns of patient selection among 820 U.S. bariatric surgical specialists was examined using clinical patient vignettes. In all BMI and comorbidity subgroups that met current NIH clinical guidelines for surgery, patient gender did not influence patient selection. This insinuates that the overrepresentation of women undergoing bariatric surgery likely occurs prior to evaluation by a bariatric surgeon and is either related to referral patterns or patient preference. However, the study did find that gender had an impact on patient selection only for the subset of patients who did not meet NIH BMI and comorbidity criteria. In this subset, men had a $67 \%$ decreased odds of selection for surgery. While the majority of patients who do not meet NIH criteria are often self-referred and appropriately excluded from surgical evaluation ${ }^{[11]}$, the findings from the Santry et al. ${ }^{[34]}$ study suggest that some surgeons may be influenced by social and cultural pressures on women to achieve body image ideals. Bariatric surgery for this subset of patients, however, likely does not completely account for the gender disparity across all bariatric surgical patients, the majority of which meet NIH criteria.

\section{SEX-BASED OUTCOMES AFTER BARIATRIC SURGERY}

Similar to the knowledge gap about the safety of weight loss surgery, differential referral for and utilization of bariatric surgery by men and women may also be founded in a lack of knowledge, on the part of both providers and patients, surrounding the effectiveness of weight loss and health risk reduction with surgery. Kennedy-Dalby et al. ${ }^{[35]}$ sought to compare sex-based outcomes in an observational cohort analysis of 79 men matched to 79 women for age, BMI, bariatric procedure, and comorbidities including type 2 diabetes and obstructive sleep apnea. At 24 months postoperatively, significant reductions in excess BMI loss were identified for both women and men ( $72.9 \%$ and $65.8 \%$, respectively). Both groups demonstrated significant reductions in hypertension, glycosylated hemoglobin, and cholesterol without significant differences by gender. Additionally, $77.5 \%$ of men and $90.0 \%$ of women with obstructive sleep apnea discontinued continuous positive airway pressure use. These findings support bariatric surgery as an effective weight loss intervention with significant improvement in metabolic and functional outcomes for both men and women.

Weight loss surgery has also been shown to have positive effects on sexual functioning, fertility, and pregnancy outcomes in women. In a prospective cohort study of 106 women who underwent bariatric surgery, Sarwer et al. ${ }^{[36]}$ reported that, within the first two postoperative years, women report significant improvements in overall sexual functioning as well as in specific domains related to arousal, lubrication, desire, and satisfaction. These observations correlated with significant improvements in levels of sex hormones as well as in important shifts across quality of life measures, including improvement in depressive symptoms, self-esteem, and overall emotional and physical functioning. Weight loss after bariatric surgery is associated with improvement in conditions such as polycystic ovarian syndrome, anovulation, and irregular menses, leading to improvement in fertility rates as well as reductions in pregnancy loss and maternal pregnancy complications such as gestational diabetes and preeclampsia ${ }^{[24]}$.

Among men pursing bariatric surgery, 36\% report sexual dissatisfaction associated with erectile dysfunction ${ }^{[23]}$. Additionally, elevated BMI has been associated with impairments in semen characteristics and reduced levels of reproductive hormones potentially affecting male fertility ${ }^{[37]}$. In a study of 97 men undergoing gastric bypass, participants reported significant improvement in sexual function based on the Brief Male Sexual Function Inventory score before and after surgery. There was improvement in all 
categories, including sexual drive ( $3.9 \pm 0.3$ to $5.3 \pm 0.3$ ), erectile function ( $6.4 \pm 0.5$ to $8.9 \pm 0.5$ ), ejaculatory function $(4.9 \pm 0.4$ to $6.3 \pm 0.4)$, problem assessment $(7.4 \pm 0.5$ to $9.6 \pm 0.5)$, and sexual satisfaction $(1.6 \pm$ 0.2 to $2.3 \pm 0.2$; all $P<0.01)^{[38]}$. Other studies have also demonstrated improvements in sexual quality of life after bariatric surgery, as well as favorable reproductive hormonal alterations in $\operatorname{men}^{[39,40]}$. The biologic (improvement in sex hormone levels) and gender-related aspects of weight loss (in part body image-related) likely work in symbiosis to create renewed vitality and a positive sense of identity for both men and women.

Vegel et al. ${ }^{[41]}$ further examined QOL outcomes after bariatric surgery at a single institution using the Moorehead-Ardelt Quality of Life Questionnaire II (MAQoLII). In total, 209 patients underwent bariatric surgery from 2010 to 2012, with 79\% being women. There was a significant improvement in scores both overall and across each category of QOL measures at one-year postoperatively compared to preoperatively, including in physical functioning, self-esteem, and sexual function. Gender was not associated with a change in outcome. In a prospective study of 32 men who underwent bariatric surgery conducted by Sarwer et al ${ }^{[42]}$, participants reported significant improvements in physical quality of life measures, weightrelated quality of life, and body image. All this proposes that, although men may not initially view the downsides of obesity on their health and functional capacity, bariatric surgery seems to significantly improve quality of life measures in both genders equally.

\section{CONCLUSION}

Bariatric surgery has evolved to become a safe and effective treatment for morbid obesity with favorable outcomes for both women and men. However, significant barriers exist between morbidly obese patients and bariatric surgical treatment, and, although these barriers exist for both men and women, there is clearly a gender disparity with far fewer eligible men receiving appropriate treatment. Thus, greater efforts are needed to improve overall access to surgical care and narrow the gender gap. This can only be accomplished through collaborations between bariatric surgical specialists and primary care providers to ensure that eligible patients receive meaningful education about the risks and benefits of surgery with attention to gendered concerns and expectations. Primary care providers, who continue to be the most important source of patient referral ${ }^{[11]}$, are in a frontline position to identify patients who may benefit from surgery and to understand patients' health-related and social values, working closely with those who may be reticent to consider bariatric surgery and less likely to independently express interest in a surgical evaluation when appropriate. Individualized multidisciplinary support remains vital throughout both the evaluation and treatment process to ensure commitment to surgery and long-term success, as surgical weight loss can truly result in profound positive changes across all aspects of life.

\section{DECLARATIONS}

\section{Authors' contributions}

Conceived the original idea: Hachey $\mathrm{K}$

Contributed to the drafting of the manuscript and critical revisions: Aly S, Hachey K, Pernar LIM

\section{Availability of data and materials}

Not applicable.

\section{Financial support and sponsorship}

None.

\section{Conflicts of interest}

All authors declared that there are no conflicts of interest.

\section{Ethical approval and consent to participate}

Not applicable. 


\section{Consent for publication}

Not applicable.

\section{Copyright}

(c) The Author(s) 2020.

\section{REFERENCES}

1. Mainous AG, Johnson SP, Saxena SK, Wright RU. Inpatient bariatric surgery among eligible black and white men and women in the United States, 1999-2010. Am J Gastroenterol 2013;108:1218-23.

2. An R. Prevalence and trends of adult obesity in the US, 1999-2012. ISRN Obes 2014;2014:185132.

3. Santry HP, Gillen DL, Lauderdale DS. Trends in bariatric surgical procedures. J Am Med Assoc 2005;294:1909-17.

4. Wang YC, Pamplin J, Long MW, Ward ZJ, Gortmaker SL, et al. Severe obesity in adults cost state medicaid programs nearly \$8 billion in 2013. Health Aff (Millwood) 2015;34:1923-31.

5. Kitahara CM, Flint AJ, Berrington de Gonzalez A, Bernstein L, Brotzman M, et al. Association between class III obesity (BMI of 40-59 $\mathrm{kg} / \mathrm{m}^{2}$ ) and mortality: a pooled analysis of 20 prospective studies. PLoS Med 2014;11:e1001673.

6. Chang SH, Stoll CRT, Song J, Varela JE, Eagon CJ, et al. The effectiveness and risks of bariatric surgery an updated systematic review and meta-analysis, 2003-2012. JAMA Surg 2014;149:275-87.

7. Puzziferri N, Roshek TB, Mayo HG, Gallagher R, Belle SH, et al. Long-term follow-up after bariatric surgery: a systematic review. JAMA 2014;312:934-42.

8. Perlman SE, Reinhold RB, Nadzam GS. How do family practitioners perceive surgery for the morbidly obese? Surg Obes Relat Dis 2007;3:428-33.

9. Nguyen NT, Masoomi H, Magno CP, Nguyen XMT, Laugenour K, et al. Trends in use of bariatric surgery, 2003-2008. J Am Coll Surg 2011;213:261-6.

10. ASMBS. Estimate of Bariatric Surgery Numbers, 2011-2018. Available from: https://asmbs.org/resources/estimate-of-bariatric-surgerynumbers [Last accessed on 24 Mar 2020]

11. Tsuda S, Barrios L, Schneider B, Jones DB. Factors affecting rejection of bariatric patients from an academic weight loss program. Surg Obes Relat Dis 2009;5:199-202.

12. Merrell J, Ashton K, Windover A, Heinberg L. Psychological risk may influence drop-out prior to bariatric surgery. Surg Obes Relat Dis 2012;8:463-9.

13. Funk LM, Jolles S, Fischer LE, Voils CI. Patient and referring practitioner characteristics associated with the likelihood of undergoing bariatric surgery a systematic review. JAMA Surg 2015;150:999-1005.

14. Wee CC, Davis RB, Chiodi S, Huskey KW, Hamel MB. Sex, race, and the adverse effects of social stigma vs. other quality of life factors among primary care patients with moderate to severe obesity. J Gen Intern Med 2015;30:229-35.

15. Fuchs HF, Broderick RC, Harnsberger CR, Chang DC, Sandler BJ, et al. Benefits of bariatric surgery do not reach obese men. J Laparoendosc Adv Surg Tech A 2015;25:196-201.

16. Gastrointestinal surgery for severe obesity: National Institutes of Health Consensus Development Conference Statement. Am J Clin Nutr 1992;55:615S-9.

17. May AL, Freedman D, Sherry B, Blanck HM; Centers for Disease Control. Obesity - United States, 1999-2010. MMWR Suppl 2013;62:120-8.

18. Flegal KM, Carroll D, Kit BK, Ogden CL. Prevalence of obesity and trends in the distribution of body mass index among US adults, 1999-2010. JAMA 2012;307:491-7.

19. Young MT, Phelan MJ, Nguyen NT. A decade analysis of trends and outcomes of male vs female patients who underwent bariatric surgery. J Am Coll Surg 2016;222:226-31.

20. Diamant A, Milner J, Cleghorn M, Sockalingam S, Okrainec A, et al. Analysis of patient attrition in a publicly funded bariatric surgery program. J Am Coll Surg 2014;219:1047-55.

21. Avidor Y, Still CD, Brunner M, Buchwald JN, Buchwald H. Primary care and subspecialty management of morbid obesity: referral patterns for bariatric surgery. Surg Obes Relat Dis 2007;3:392-407.

22. Balduf LM, Farrell TM. Attitudes, beliefs, and referral patterns of PCPs to bariatric surgeons. J Surg Res 2008;144:49-58.

23. Sarwer DB, Spitzer JC, Wadden TA, Rosen RC, Mitchell JE, et al. Sexual functioning and sex hormones in persons with extreme obesity and seeking surgical and nonsurgical weight loss. Surg Obes Relat Dis 2013;9:997-1007.

24. Alatishe A, Ammori BJ, New JP, Syed AA. Bariatric surgery in women of childbearing age. QJM 2013;106:717-20.

25. Tsai SA, Lv N, Xiao L, Ma J. Gender differences in weight-related attitudes and behaviors among overweight and obese adults in the United States. Am J Mens Health 2016;10:389-98.

26. White MA, O’Neil PM, Kolotkin RL, Byrne TK. Gender, race, and obesity-related quality of life at extreme levels of obesity. Obes Res 2004; $12: 949-55$.

27. Wee CC, Huskey KW, Bolcic-Jankovic D, Colten ME, Davis RB, et al. Sex, race, and consideration of bariatric surgery among primary care patients with moderate to severe obesity. J Gen Intern Med 2014;29:68-75.

28. Libeton M, Dixon JB, Laurie C, O'Brien PE. Patient motivation for bariatric surgery: characteristics and impact on outcomes. Obes Surg 2004; $14: 392-8$

29. Wee CC, Jones DB, Davis RB, Bourland AC, Hamel MB. Understanding patients' value of weight loss and expectations for bariatric surgery. Obes Surg 2006;16:496-500. 
30. Natvik E, Gjengedal E, Moltu C, Råheim M. Translating weight loss into agency: men's experiences 5 years after bariatric surgery. Int J Qual Stud Health Well-being. 2015;10:277729.

31. Funk LM, Jolles SA, Voils CI. Obesity as a disease: has the AMA resolution had an impact on how physicians view obesity? Surg Obes Relat Dis 2016;12:1431-5.

32. Funk LM, Jolles SA, Greenberg CC, Schwarze ML, Safdar N, et al. Primary care physician decision making regarding severe obesity treatment and bariatric surgery: a qualitative study. Surg Obes Relat Dis 2016;12:893-901.

33. Aminian A, Brethauer SA, Kirwan JP, Kashyap SR, Burguera B, et al. How safe is metabolic/diabetes surgery? Diabetes Obes Metab 2015;17:198-201.

34. Santry HP, Lauderdale DS, Cagney KA, Rathouz PJ, Alverdy JC, et al. Predictors of patient selection in bariatric surgery. Ann Surg 2007;245:59-67.

35. Kennedy-Dalby A, Adam S, Ammori BJ, Syed AA. Weight loss and metabolic outcomes of bariatric surgery in men versus women - a matched comparative observational cohort study. Eur J Intern Med 2014;25:922-5.

36. Sarwer DB, Spitzer JC, Wadden TA, Mitchell JE, Lancaster K, et al. Changes in sexual functioning and sex hormone levels in women following bariatric surgery. JAMA Surg 2014;149:26-33.

37. Andersen JM, Herning H, Aschim EL, Hjelmesæth J, Mala T, et al. Body mass index is associated with impaired semen characteristics and reduced levels of anti-Müllerian hormone across a wide weight range. PLoS One 2015;10:e0130210.

38. Dallal RM, Chernoff A, O'Leary MP, Smith JA, Braverman JD, et al. Sexual dysfunction is common in the morbidly obese male and improves after gastric bypass surgery. J Am Coll Surg 2008;207:859-64.

39. Hammoud A, Gibson M, Hunt SC, Adams TD, Carrell DT, et al. Effect of roux-en-Y gastric bypass surgery on the sex steroids and quality of life in obese men. J Clin Endocrinol Metab 2009;94:1329-32.

40. Mora M, Aranda GB, De Hollanda A, Flores L, Puig-Domingo M, et al. Weight loss is a major contributor to improved sexual function after bariatric surgery. Surg Endosc 2013;27:3197-204.

41. Vegel AJ, Shah N, Lidor AO, Greenberg JA, Shan Y, et al. Patient-reported quality of life after bariatric surgery: a single institution analysis. J Surg Res 2017;218:117-23.

42. Sarwer DB, Spitzer JC, Wadden TA, Rosen RC, Mitchell JE, et al. Sexual functioning and sex hormones in men who underwent bariatric surgery. Surg Obes Relat Dis 2015;11:643-51. 